\title{
Morfologia externa do adulto de Almeidaia aidae Mielke \& Casagrande (Lepidoptera, Saturniidae, Arsenurinae, Almeidaiini). I. Cabeça, apêndices e região cervical ${ }^{1}$
}

\author{
Amabílio J. A. de Camargo ${ }^{2}$, Mirna M. Casagrande ${ }^{3}$, Olaf H. H. Mielke ${ }^{3} \&$ Eurides Furtado ${ }^{4}$ \\ ${ }^{1}$ Contribuição número 1539 do Departamento de Zoologia, Universidade Federal do Paraná. \\ ${ }^{2}$ Embrapa Cerrados. Rodovia BR 020, Km 18, Caixa Postal 08223, 73310-970 Planaltina, Distrito Federal, Brasil. \\ ${ }^{3}$ Departamento de Zoologia, Universidade Federal do Paraná. Caixa Postal 19020, 81531-980 Curitiba, Paraná, Brasil. \\ Pesquisador CNPq. E-mail: omhesp@ufpr.br; mibras@ufpr.br \\ ${ }^{4}$ Caixa Postal 97, 78400-000 Diamantino, Mato Grosso, Brasil.
}

\begin{abstract}
External morphology of Almeidaia aidae Mielke \& Casagrande (Lepidoptera, Saturniidae, Arsenurinae, Almeidaiini). I. Head, appendages and cervical region. The external morphology of adult head of Almeidaia aidae Mielke \& Casagrande, 1981 is described and illustrated for the first time. The results obtained were compared with other species of Saturniidae. This species is rare and endemic of the Cerrado region. Biological studies were published recenthly by the fourth author.
\end{abstract}

KEY WORDS. Brazil, morphology, moth, Neotropical.

RESUMO. A morfologia externa do adulto de Almeidaia aidae Mielke \& Casagrande, 1981 é descrita e ilustrada pela pela primeira vez. Os resultados obtidos foram comparados com outras espécies de Saturniidae. É uma espécie rara e endêmica da região do Cerrado. Estudos sobre sua biologia foram publicados recentemente pelo quarto autor. PALAVRAS CHAVE. Brasil, morfologia, mariposa, Neotropical.

Almeidaia aidae Mielke \& Casagrande, 1981 é o menor Arsenurinae conhecido. A espécie foi descrita com base em um casal da coleção Gagarin, atualmente no Departamento de Zoologia da Universidade Federal do Paraná. Os exemplares foram coletados em Rio Verde, Mato Grosso do Sul pelo Sr. Pedro Pompeu da Silva. GAGARIN (1966) identifica estes exemplares como sendo Almeidaia romualdoi Travassos, 1937, designando a fêmea como alótipo. Outro casal, de mesma procedência, foi igualmente identificado e ilustrado por Lemaire (1980). Dados das etiquetas do holótipo macho: /Ex col. Gagarin/Holótipo/XI-1966 Rio Verde, M.T., A. Maller leg/Gen. prep. Mielke 1980/Almeidaia aidae Mielke \& Casagrande. Mielke \& Casagrande det. 1980, Holótipo/DZ 2489/. Dados das etiquetas para o alótipo fêmea: /Ex Col. Gagarin/ Almeidaia romualdoi Alótipo/10-1962 Rio Verde, M.T./Gen. prep. Mielke 1980/ Almeidaia romualdoi Travassos, 1937, Gagarin det. 1966, Alótipo/Parátipo Almeidaia aidae/Parátipo Almeidaia aidae Mielke \& Casagrande. Mielke \& Casagrande det. 1980/DZ 2490/. A informação A. Maller leg. na etiqueta do holótipo indica que o exemplar veio através deste fornecedor, falecido alguns anos atrás em Corupá, Santa Catarina, cidade em que residia. Na época, Rio Verde pertencia ao Estado de Mato Grosso (MT), hoje desmembrado em Mato Grosso (MT) e Mato Grosso do Sul (MS).

Desde sua descrição, a espécie tem sido pouco coletada e estudada, e as informações disponíveis são apenas aquelas publicadas na descrição original de MielKe \& CASAGRANDE (1981), onde são apresentadas fotografias dos adultos e ilustrações de venação e genitália. No entanto, mais recentemente, vários exemplares foram coletados e criados em Diamantino, MT pelo quarto autor, tornando possível estudos mais detalhados de morfologia e biologia (FURTADO 2004).

Este é o primeiro de três artigos sobre a morfologia externa da espécie. O presente objetiva esclarecer a morfologia externa da cabeça, apêndices e região cervical.

\section{MATERIAL E MÉTODOS}

O material estudado é proveniente de coleta de formas imaturas e criação até a obtenção de adultos, realizada pelo quarto autor em Diamantino, MT.

Os exemplares foram fervidos em solução de $\mathrm{KOH} 10 \%$ (hidróxido de potássio) em banho-maria durante 10 minutos, com posterior remoção da cabeça, apêndices e esclerito cervical. As ilustrações foram feitas com auxílio de microscópio estereoscópico e câmara clara acoplada. Para a conservação das estruturas foi utilizado GFA, líquido preparado conforme descrito em CASAGRANDE (1979). A escala para cada desenho representa $1 \mathrm{~mm}$.

Quanto à nomenclatura utilizada para a morfologia, existem divergências na literatura, as quais foram adequadamente discutidas por Mielke et al. (2004). Nesse estudo optou-se preferencialmente pelo uso de terminologia específica para Saturniidae, como em Michener (1952) e Lemaire (1971). Em alguns casos utilizou-se Beutelspacher (1967), Casagrande (1979), Duarte et al. (2001), Bizarro et al. (2003) e MielKe et al. (2004). 


\section{RESULTADOS E DISCUSSÃO}

Estudos morfológicos completos sobre as espécies da família Saturniidae são escassos na literatura. Na maioria das vezes é ilustrada apenas a genitália, e em alguns casos a venação. Assim, a morfologia de Almeidaia aidae foi comparada, em seus aspectos gerais, com Eacles imperialis (Drury, 1773) (Ceratocampinae) estudada por Michener (1952) e com Automeris postalbida Schaus, 1900 (Hemileucinae) por Lemaire (1971).

\section{Cabeça (Figs 1-9)}

Com exceção das antenas, outras estruturas na cabeça não apresentam dimorfismo sexual. Hipognata, mais larga do que longa, ocelos ausentes, olhos compostos esféricos, ocupando a maior parte da cabeça. Frontoclípeo semitriangular, mais estreito na região inferior, delimitado na parte superior pela sutura transfrontal, lateralmente pela área paraocular e inferiormente pela sutura clípeo-labral e fossas tentoriais anteriores. Área paraocular estreita, abaixo do rudimento mandibular. A fossa tentorial anterior apresenta-se como uma invaginação medianamente esclerotinizada no exoesqueleto, localizada na extremidade da sutura clípeo-labral. Vértice fortemente destacado, delimitado pelos alvéolos antenais e sutura transfrontal. Em vista anterior (Fig.1) observam-se ainda a inserção das antenas, escapo e pedicelo, labro, epifaringe, palpos labiais e rudimento mandibular, descritos e discutidos separadamente como apêndices cefálicos.

Em vista dorsal destaca-se o vértice, delimitado anteriormente pelo frontoclípeo e posteriormente pela sutura pós-occipital. Imediatamente após esta, uma estreita barra, denominada pós-occipicio, contorna o forame occipital (Fig. 2).

$\mathrm{Na}$ face posterior (Fig. 3) destaca-se o forame occipital, dividido em duas cavidades, pela ponte tentorial, os foramens superior e inferior. A ponte tentorial é composta por uma barra transversal estreita, fortemente esclerotinizada, e por prolongamentos projetados ventral e dorsalmente, em forma de cruz que se prolonga até cerca da metade do forame occipital, está ausente em E. imperialis, enquanto que em $A$. postalbida é uma estrutura longa que se estende desde a ponte tentorial até a face anterior do forame.

Côndilos occipitais, onde se articulam os escleritos cervicais, aparecem como expansões laterais da ponte tentorial. O forame superior, circular de diâmetro maior que o inferior, está delimitado dorsalmente pelo pós-occipício, lateralmente pela pós-gena e na sua parte inferior pela ponte tentorial. Por sua vez, o forame inferior, semipentagonal e de menor diâmetro, está limitado dorsalmente pela ponte tentorial, lateralmente pela pós-gena e ventralmente pela epifaringe.

Pós-occipício fortemente destacado e definido pela sutura pós-occipital semi esclerotinizada, especialmente na área próxima à ponte tentorial. A pós-gena, composta de duas áreas, uma mais escura, estreita e externa e outra larga e mais clara, está delimitada pelo forame occipital e pela área occipital. Esta, por sua vez, apresenta também cor clara e é delimitada pelo olho composto e pela porção mais escura e estreita da pós-gena.
Os Saturniidae, como os demais Bombycoidea, apresentam ausência ou redução das estruturas do aparelho bucal. Nessa espécie consistem apenas de rudimentos mandibulares, gáleas, estipes, lábio e palpos labiais que ocupam a face ventral da cabeça entre as áreas paraoculares (Fig. 4). Os rudimentos mandibulares e o lábio aparecem como uma área plana na região média posterior nas espécies comparadas. Lateralmente, na parte inferior, o frontoclípeo e os palpos labiais triarticulados são proeminentes (Fig. 5).

\section{Apêndices cefálicos \\ Antenas (Figs 6 e 7)}

Localizadas em disposição dorso-lateral em relação ao frontoclípeo e separadas na base pela sutura transfrontal. Do tipo dentada nos machos e filiforme nas fêmeas. Compostas de um artículo basal, escapo, de forma cilíndrica e consideravelmente maior que o segundo, pedicelo, com forma anelar. Os demais artículos constituem o flagelo. Nos machos examinados encontrou-se 43 antenômeros providos de cílios sensitivos, sensilas, laterais e apicais, mais abundantemente distribuídos nos antenômeros basais e medianos. Nas fêmeas, 44 antenômeros desprovidos de sensilas.

O padrão de antenas de $A$. aidae segue aquele encontrado na maioria das espécies de Saturniidae, que apresentam antenas de maneira geral, denteadas ou pectinadas nos machos e filiformes na maioria das fêmeas, nestas raramente denteadas ou pectinadas. No entanto, as espécies da subfamília Ceratocampinae constituem-se em exceção, pois apresentam antenas mistas, isto é, com terços basal e mediano modificados em relação ao terço apical, caráter mais evidente nos machos.

\section{Labro (Fig. 1)}

Estrutura larga, transversal, horizontalmente bicarenada e pouco esclerotinizada e separado do frontoclípeo pela sutura clípeo-labral. No centro, a epifaringe está representada por um pequeno lobo triangular. Ao contrário de outros Arsenurinae mencionados por Lemaire (1980), não foram observados pilíferos, par de estruturas situadas em posição látero-dorsal às gáleas, reduzidas em $A$. aidae e $A$. postalbida, e mais desenvolvidas em $E$. imperialis.

Pilíferos podem ser vestigiais em muitas espécies, e sua presença é mais facilmente visualizada quando acompanhada de pêlos. Estes pêlos estão ausentes nas espécies das subfamílias Hemileucinae, Saturniinae e Ceratocampinae, mas presentes nos Arsenurinae (Lemaire 1971, 1978, 1980, 1988). A ausência dessa estrutura em $A$. aidae consiste em uma diferença significativa para com os demais Arsenurinae.

\section{Maxilas (Fig. 4)}

Localizadas na porção anterior da fossa proboscidial, constituídas por um par de gáleas rudimentares sustentadas pelos estipes, estes proeminentes, esclerotinizados e projetados para a região anterior da fossa. Em outros Arsenurinae, como certas espécies de Arsenura, as gáleas são mais proeminentes, apesar de pouco desenvolvidas. Esta característica, aliada a outras, auxilia 

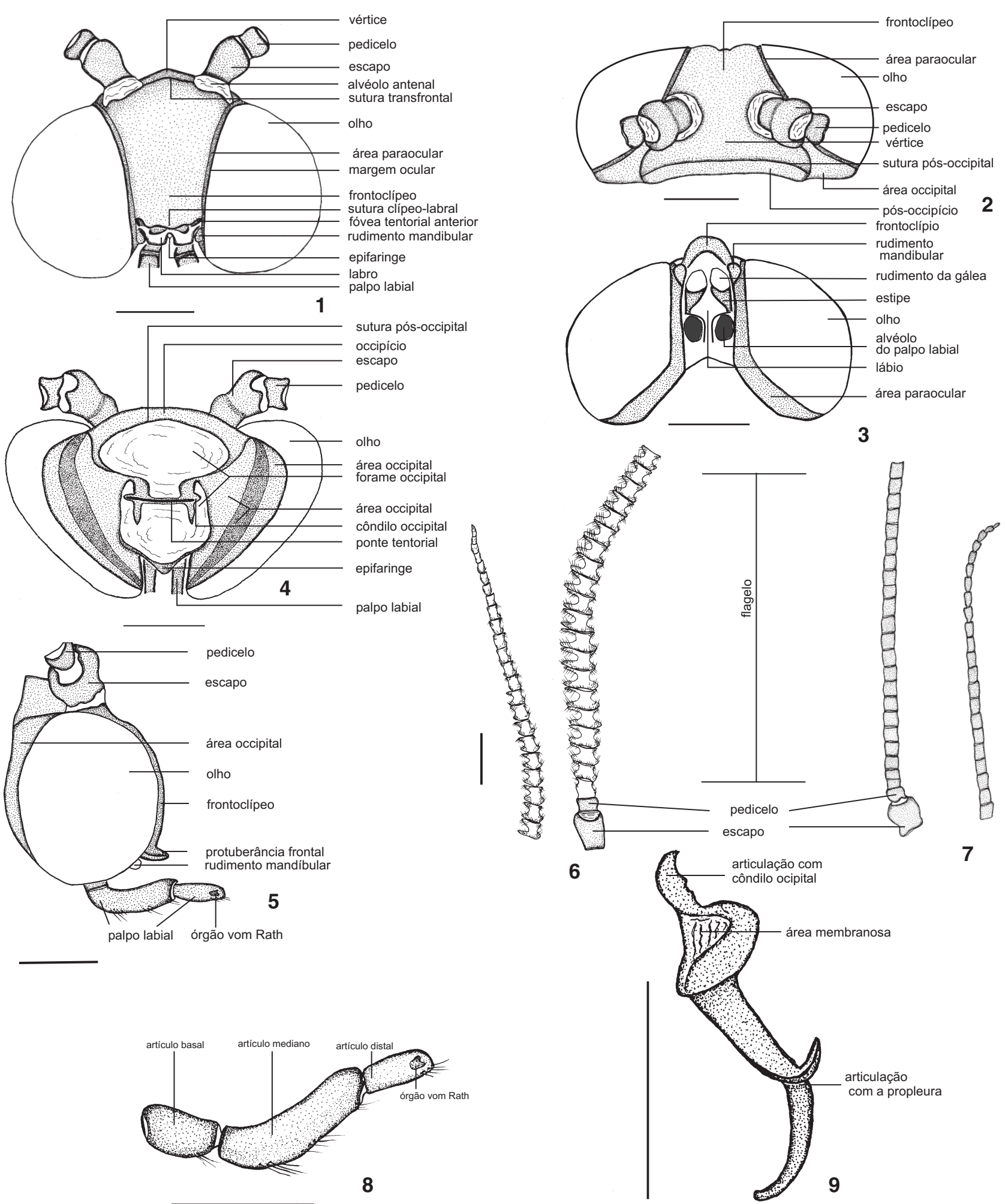

Figuras 1-9. (1-5) Cabeça vista: (1) anterior, (2) dorsal, (3) posterior, (4) ventral, (5) lateral; (6-7) antenas: (6) macho, (7) fêmea; (8) palpo labial esquerdo, vista lateral, interna; (9) esclerito cervical, vista lateral. 
na identificação das tribos Arsenurini e Almeidaiini. Palpos maxilares e cardos ausentes.

\section{Lábio (Figs 4 e 8)}

Apresenta-se como uma área plana na região mediana ventral e posterior da fossa proboscidial. Anteriormente estão as gáleas rudimentares, e na posição látero-posterior a inserção dos palpos labiais, circundados por uma acentuada depressão (Fig. 4).

Palpos labiais (Fig. 8) compostos por três artículos cilíndricos com cerdas. Artículo basal curto, com menos de um terço do comprimento total do palpo e inserido no esclerito labial. Artículo mediano maior, cerca de metade do tamanho total do palpo, e com espessura aproximadamente igual ao do artículo basal. $\mathrm{O}$ artículo distal, de tamanho e espessura inferiores aos anteriores, apresenta na porção distal mancha sensitiva denominada Órgão “vom Rath" (SCOBLE 1995). Em relação às espécies que foram utilizadas nas comparações, o palpo labial também é triarticulado, com exceção de E. imperialis que possui dois artículos com tamanhos semelhantes. Outras espécies das subfamílias Saturniinae, Ceratocampinae e Hemileucinae podem apresentar dois ou três artículos (Lemarre 1978, 1988, 2002). Em Rothschildia jacobaeae (Walker, 1855) (Saturniinae), por exemplo, os palpos labiais são semelhantes aos de Arsenurinae, isto é, tri-articulados e de tamanhos diferentes entre si. Em Oxyteninae, são biarticulados, com o artículo basal longo e o apical reduzido.

\section{Esclerito cervical (Fig. 9)}

A cabeça une-se ao tórax através da região cervical, quase exclusivamente membranosa, exceto pelo par de escleritos cervicais, que se articulam anteriormente com os côndilos occipitais e posteriormente com as propleuras. Apresentam-se fortemente esclerotinizados, com uma área membranosa na porção anterior, uma área mediana com leve depressão central e uma posterior afilada e curva que, próxima à articulação com a propleura, apresenta ainda uma projeção inferior levemente curva e estreita.

\section{AGRADECIMENTOS}

À Empresa Brasileira de Pesquisa Agropecuária EMBRAPA, especialmente ao Centro de Pesquisa Agropecuária dos Cerrados - CPAC, que proporcionou ao autor sênior os meios para execução do trabalho. À bióloga Karen Schmidt pelo apoio e sugestões. Aos técnicos Wellington Cavalcanti pelo auxílio com o programa de tratamento das figuras, e Jânio Fonseca Silva nos trabalhos de laboratório.

\section{REFERÊNCIAS BIBLIOGRÁFICAS}

Beutelspacher, C. 1967. Estudio morfologico de Erinnys ello (Linnaeus, 1758) (Lepidoptera, Sphingidae). Anales del Ins- tituto de Biología de la Universidad Nacional Autónoma de México, Série Zoología, Ciudad de México, 38 (1): 59-74.

Bizarro, J.M.S.; M.M. Casagrande \& O.H.H. MielKe. 2003. Morfologia externa de Thyridia psidii cetoides (Rosenberg \& Talbot). I. Cabeça e apêndices (Lepidoptera, Nymphalidae, Ithomiinae). Revista Brasileira de Zoologia, Curitiba, 20 (2): 279284.

Casagrande, M.M. 1979. Sobre Caligo beltrao (Illiger).II: Morfologia externa da cabeça do adulto (Lepidoptera, Satyridae, Brassolinae). Revista Brasileira de Biologia, Rio de Janeiro, 39 (1): 223-227.

Duarte, M.; M.M. Casagrande \& O.H.H. Mielke. 2001. Morfologia externa do adulto de Hemiargus hanno (Stoll) (Lepidoptera, Lycaenidae, Polyommatinae, Polyommatini). I. Cabeça. Revista Brasileira de Zoologia, Curitiba, 18 (1): 225-238.

FurTado, E. 2004. Almeidaia aidae Mielke \& Casagrande: seus estágios imaturos e notas taxonômicas (Lepidoptera, Saturniidae, Arsenurinae, Almeidaiini). Revista Brasileira de Zoologia, Curitiba, 21 (3): 663-669.

Gagarin, P. 1966. Sobre o exemplar feminino de "Almeidaia romualdoi" Travassos, 1937. Atas da Sociedade de Biologia do Rio de Janeiro 10 (4): 95.

Lemaire, C. 1971. Révision du genre Automeris Hübner et des genres voisins. Biogéographie, éthologie, morphologie, taxonomie (Lep. Attacidae). Mémoires du Muséum National D'Histoire Naturelle, Paris, 68: 1-576.

Lemaire, C. 1978. Les Attacidae Américains ( = Saturniidae). Attacinae. Édition C. Lemaire, Neuilly-sur-Seine, 238p.

Lemaire, C. 1980. Les Attacidae Américains ( = Saturniidae). Arsenurinae. Édition C. Lemaire, Neuilly-sur-Seine, 199p.

Lemaire, C. 1988. Les Attacidae Américains ( = Attacidae). Ceratocampinae. Museo Nacional de Costa Rica. San José, Costa Rica. 480p.

Lemaire, C. 2002. The Attacidae of America. Hemileucinae. Part A. Goecke \& Evers, Keltern, Germany, 1-688p.

Michener, C.D. 1952. The Saturniidae (Lepidoptera) of the Western Hemisphere, morphology, phylogeny, and classification. Bulletin of the American Museum of Natural History, New York, 98 (5): 335-502.

Mielke, C.G.C.; O.H.H. Mielke \& M.M. Casagrande. 2004. Estudo comparado da morfologia externa de Zaretis itys itylus (Westwood) e Agrias claudina annetta (Gray) (Lepidoptera, Nymphalidae, Charaxinae). I. Cabeça, apêndices e região cervical. Revista Brasileira de Zoologia, Curitiba, 21 (2): 357-369.

Mielke, O.H.H \& M.M. Casagrande. 1981. Notas sobre o gênero Almeidaia Travassos, com descrição de uma espécie nova (Lepidoptera, Saturniidae, Arsenurinae). Revista Brasileira de Biologia, Rio de Janeiro, 41 (4): 809-813.

Scoble, M.J. 1995. The Lepidoptera: form, function, and diversity. New York, Oxford University Press, XII+404p.

Recebido em 24.IX.2004; aceito em 01.VIII.2005.

Revista Brasileira de Zoologia 22 (3): 619-622, setembro 2005 